\title{
Novel Features of Cryptochrome-Mediated Photoreception in the Brain Circadian Clock of Drosophila
}

\author{
André Klarsfeld, Sébastien Malpel, Christine Michard-Vanhée, Marie Picot, Elisabeth Chélot, and François Rouyer \\ Institut de Neurobiologie Alfred Fessard, Centre National de la Recherche Scientifique, Unité Propre de Recherche 2216, 91198 Gif-sur-Yvette, France
}

\begin{abstract}
In Drosophila, light affects circadian behavioral rhythms via at least two distinct mechanisms. One of them relies on the visual phototransduction cascade. The other involves a presumptive photopigment, cryptochrome (cry), expressed in lateral brain neurons that control behavioral rhythms. We show here that cry is expressed in most, if not all, larval and adult neuronal groups expressing the PERIOD (PER) protein, with the notable exception of larval dorsal neurons (DN2s) in which PER cycles in antiphase to all other known cells. Forcing cry expression in the larval DN2s gave them a normal phase of PER cycling, indicating that their unique antiphase rhythm is related to their lack of cry expression. We were able to directly monitor CRY protein in Drosophila brains in situ. It appeared highly unstable in the light, whereas in the dark, it accumulated in both the nucleus and the cytoplasm, including some neuritic projections. We also show that dorsal PER-expressing brain neurons, the adult DN1s, are the only brain neurons to coexpress the CRY protein and the photoreceptor differentiation factor GLASS. Studies of various visual system mutants and their combination with the $c r y^{b}$ mutation indicated that the adult DN1s contribute significantly to the light sensitivity of the clock controlling activity rhythms, and that this contribution depends on CRY. Moreover, all CRY-independent light inputs into this central behavioral clock were found to require the visual system. Finally, we show that the photoreceptive DN1 neurons do not behave as autonomous oscillators, because their PER oscillations in constant darkness rapidly damp out in the absence of pigment-dispersing-factor signaling from the ventral lateral neurons.
\end{abstract}

Key words: locomotor activity rhythms; entrainment; circadian photoreception; cryptochrome; GLASS protein; PERIOD protein; pigment-dispersing factor; dorsal neurons

\section{Introduction}

Circadian clocks, found in virtually all living organisms, keep running in constant conditions with species-specific periods of circa $24 \mathrm{hr}$. They are synchronized by the diurnal cycling in light intensity. Short nighttime light pulses phase-shift the clock by several hours, whereas constant light ( $>1-10$ lux in Drosophila) usually suppresses free-running rhythmicity altogether (Aschoff, 1979; Konopka et al., 1989). In Drosophila, behavioral rhythms are primarily controlled by a small set of brain neurons (HelfrichFörster, 1998; Renn et al., 1999; Blanchardon et al., 2001), the

Received Aug. 5, 2003; revised Dec. 10, 2003; accepted Dec. 11, 2003.

This work was supported by grants from Centre National de la Recherche Scientifique (CNRS) (Action Thématique Incitative sur Programme et Équipes "Développement" and appel d'offres "Biologie cellulaire"), Fondation pour la Recherche Médicale (FRM), and Ministère de la Recherche Action Concertée Incitative (Biologie du développement et physiologie intégrative). S.M. was supported successively by Ministère de l'Education Nationale, de la Recherche et de la Technologie, FRM, and the Association pour la Recherche sur le Cancer, and F.R. was supported by Institut National de la Santé et de la Recherche Médicale. The mAb9B2.1 developed by G. Rubin was obtained from the Developmental Studies Hybridoma Bank (University of lowa). M. Ahmad (Université Paris 6) provided the neutral density and red filters and photometer as well as many illuminating discussions. We thank A. Bergmann, P. Emery, J. Hall, A. Hofbauer, R. Stanewsky, and the Bloomington Drosophila Stocks Center for providing fly strains and antibodies. We are grateful to S. Brown and C. Talbot (Institut des Sciences Végetales, CNRS, Gif-sur-Yvette) for help with confocal imaging, J. Kouévi for contributing to the characterization of the cry-gal4 lines, M. Boudinot for the Faas software, G. Levesque and J.-Y. Tiercelin for help with the behavioral analysis set-up, L. Collet for artwork, and B. Grima and A. Lamouroux for critical reading of this manuscript.

Correspondence should be addressed to François Rouyer, Institut de Neurobiologie Alfred Fessard, Centre $\mathrm{Na}$ tional de la Recherche Scientifique Unité Propre de Recherche 2216 Avenue de la Terrasse, 91198 Gif-sur-Yvette Cedex, France. E-mail: rouyer@iaf.cnrs-gif.fr.

DOI:10.1523/JNEUROSCI.3661-03.2004

Copyright $\odot 2004$ Society for Neuroscience $\quad$ 0270-6474/04/241468-10\$15.00/0 ventral lateral neurons $\left(\mathrm{LN}_{\mathrm{v}} \mathrm{s}\right)$. These cells express the pigmentdispersing-factor (PDF), a neuropeptide required for robust rhythmicity (Helfrich-Förster, 1995; Renn et al., 1999). In addition to the $\mathrm{LN}_{\mathrm{v}} \mathrm{s}$, four other neuronal groups in the dorsolateral $\left(\mathrm{LN}_{\mathrm{d}} \mathrm{s}\right)$ and dorsal (DN1s, DN2s, and DN3s) brain express PERIOD (PER) cyclically (Kaneko et al., 1997; Kaneko, 1998; Blanchardon et al., 2001). Their contribution to behavioral rhythmicity is unknown. In the larval brain, cyclic PER expression is observed in five LNs and two DN1s, whereas two DN2s display antiphasic PER oscillations (Kaneko et al., 1997). The adult $\mathrm{LN}_{\mathrm{v}} \mathrm{s}$ are closely associated to the medulla of the optic lobe (Helfrich-Förster and Homberg, 1993) and to extraretinal photoreceptors known as the Hofbauer-Buchner (HB) eyelet (Hofbauer and Buchner, 1989; Malpel et al., 2002). Functional connections between the $\mathrm{LN}_{\mathrm{v}} \mathrm{s}$ and the eyes are strongly inferred from the higher light intensities needed to entrain visual system mutants (Wheeler et al., 1993; Stanewsky et al., 1998; HelfrichFörster et al., 2002).

Visually blind mutants still respond to light via cryptochrome (CRY) (Emery et al., 1998; Stanewsky et al., 1998; Hall, 2000), a protein originally described in plants as a blue-light photoreceptor (Ahmad, 1999). Drosophila $\mathrm{cry}^{b}$ mutants are behaviorally rhythmic, but all of their circadian light responses are either reduced or eliminated (Stanewsky et al., 1998; Emery et al., 2000a). CRY is also involved in light input into the larval clock, because only larvae lacking both CRY and the visual system are circadianly blind (Kaneko et al., 2000; Malpel et al., 2004). Indeed, light- 
Table 1. Effect of constant light on locomotor activity rhythms

\begin{tabular}{|c|c|c|c|c|c|c|c|c|}
\hline Genotype & $500 \operatorname{lux}^{a}$ & & $50 \operatorname{lux}$ & & $5 \operatorname{lux}$ & & $0.5 \operatorname{lux}$ & \\
\hline$C S$ & $33 \pm 15 \%$ & $24.2 \pm 1.8$ & $30 \pm 0 \%$ & $22.9 \pm 0.6$ & $38 \pm 2 \%$ & $23.5 \pm 0.6$ & $86 \pm 3 \%$ & $23.8 \pm 0.3$ \\
\hline$w$ & $26 \pm 12 \%$ & $24.1 \pm 0.6$ & $42 \%^{b}$ & $25.6 \pm 0.4$ & $50 \%$ & $24.8 \pm 0.5$ & ND & ND \\
\hline$c r y^{b} s s$ & $98 \pm 3 \%$ & $25.4 \pm 0.1$ & $85 \pm 15 \%$ & $25.0 \pm 0.5$ & $75 \pm 12 \%$ & $24.0 \pm 0.3$ & $85 \pm 5 \%$ & $23.4 \pm 0.3$ \\
\hline w;GMR-hid & $21 \pm 5 \%$ & $24.4 \pm 0.9$ & $15 \%$ & $27.3 \pm 1.8$ & $20 \%$ & $24.7 \pm 0.7$ & $20 \%$ & $26.7 \pm 1.0$ \\
\hline$w ; g l^{60 J}$ & $73 \pm 6 \%$ & $26.2 \pm 0.2$ & $74 \pm 5 \%$ & $26.2 \pm 0.2$ & $84 \pm 4 \%$ & $27.0 \pm 0.2$ & $79 \pm 13 \%$ & $25.6 \pm 0.9$ \\
\hline$w n o r p A^{p 24}$ & $4 \pm 4 \%$ & $26.5^{c}$ & $15 \%$ & $22.5 \pm 1.5$ & $4 \pm 4 \%$ & $14.5^{c}$ & $0 \%{ }^{d}$ & \\
\hline
\end{tabular}

Percentage of rhythmic flies and their mean period are given for four different light intensities in LL. Rhythmicity was assessed from periodogram analysis for flies kept in LL for at least $7 \mathrm{~d}$. The percentage of rhythmic flies and their period (in hours) are generally means \pm SEM of two to five independent experiments, each with 8-25 active flies for each genotype and light intensity.

a Light intensity experienced by individual flies varied by less than a factor of two around the values shown.

${ }^{b}$ Rhythmicity values without an SEM are single experiments.

'Period of a single rhythmic fly.

${ }^{d}$ Five of these eight flies were subsequently rhythmic in constant darkness. ND, Not determined.

activated CRY affects the stability of the timeless (TIM) protein and is subsequently degraded (Stanewsky et al., 1998; Ceriani et al., 1999; Ivanchenko et al., 2001; Lin et al., 2001). All behavioral phenotypes of the $c r y^{b}$ mutant were rescued by targeted CRY expression in the $\mathrm{LN}_{\mathrm{v}} \mathrm{s}$, but rescue was more complete with a wider CRY expression, suggesting it may also play a role outside the $\mathrm{LN}_{\mathrm{v}} \mathrm{s}$ (Emery et al., 2000b). Combining $c r y^{b}$ with the $g l^{60 J}$ mutation, which removes the GLASS photoreceptor differentiation factor (Moses et al., 1989), completely blinds the behavioral clock (Helfrich-Förster et al., 2001). The $g l^{60 J}$ mutant lacks not only the retinal photoreceptors and the $\mathrm{HB}$ eyelet but also the adult DN1 set of dorsal PER-expressing neurons. These DN1s project toward the $\mathrm{LN}_{\mathrm{v}} \mathrm{s}$ (Kaneko and Hall, 2000) and could have provided a novel type of light input to the clock.

Here, we determine the expression profile of CRY protein in the Drosophila brain. We show that forcing CRY expression in a subset of larval dorsal neurons, the DN2s, which are lacking in it, changes their phase of PER cycling. Our behavioral and molecular data indicate a significant cry-dependent contribution of the adult DN1s to the photosensitivity of the brain clock.

\section{Materials and Methods}

Strains and fly rearing. Control strains were laboratory stocks of Canton-S (CS), $y w$, and $w$ flies, which behaved similarly. The $g l^{60 J}$ mutation, which prevents all photoreceptor differentiation (Moses et al., 1989), was back-crossed several times into our $w$ background. The crygal4 lines used in this study were new insertions derived from the published cry-gal4 line, which revealed only the adult $\mathrm{LN}_{\mathrm{v}} \mathrm{s}$ and probably the $\mathrm{LN}_{\mathrm{d}} \mathrm{s}$ (Emery et al., 2000b). The new insertions were produced by P-element jumping and selected for driving stronger green fluorescent protein (GFP) expression from the upstream activating sequence (UAS). When analyzed as double homozygotes with the UAS-gfp reporter, the overall expression pattern of all cry-gal4 insertions was similar (data not shown) and was not limited to the canonical clock cells, as also reported for the intronless cry promoter used by Zhao et al. (2003). The w; GMRhid strain (Bergmann et al., 1998) expresses the apoptosis gene hid under control of a glass-multimer-response (GMR) element. This multimer of a GLASS-binding site present in the $R h 1$ promoter is much more active in the eye disk than in other GLASS-expressing cells (Ellis et al., 1993; Hay et al., 1994; our unpublished results). The compound eyes of GMR-hid heterozygote flies are much reduced (Bergmann et al., 1998). In homozygotes, they were even less visible than those of $g l^{60 J}$ mutants (data not shown). No retinular or ocellar fibers were observed in adult GMR-hid homozygotes with an anti-chaoptin antibody or in adult GMR-hid heterozygotes with a Rh6-gfp reporter gene (data not shown). The complete disappearance of all photoreceptor cell bodies expressing hid from Rh1 promoter sequences has been similarly reported recently (Hsu et al., 2002). w norpA $A^{p 24}$ (Pearn et al., 1996), yw; cry ${ }^{b}$ ss (Emery et al., 1998; Stanewsky et al., 1998), $y w ; g^{60 J} \mathrm{cry}^{b}$ (Helfrich-Förster et al., 2001), $y w$; $p d f^{01}$ (Renn et al., 1999), yw; tim-gal4 (Kaneko, 1998), and $y w$; UAS-cry (Emery et al., 2000b) flies have been described previously. The $c r y^{b}$ ss chromosome was also introduced into a $w$ background as well as into our standard CS background. All measured phenotypes, notably behavioral rhythmicity and period in constant light (LL), were similar to those of the original strain (see Table 2, and data not shown). w; pdf $f^{01}$, w; GMR-hid; $c r y^{b} s s$, and $w$ norp $A^{p 24} ; c^{b}{ }^{b}$ ss flies were obtained through standard crosses. Drosophila cultures were usually maintained on a $12 \mathrm{hr}$ light/dark cycle on standard corn meal-yeast-agar medium at $25^{\circ} \mathrm{C}$ and $50 \%$ relative humidity.

Behavioral analyses. The locomotor activity of the flies was followed with commercially available monitors (TriKinetics, Waltham, MA) and analyzed with the Faas software as described previously (Blanchardon et al., 2001; Klarsfeld et al., 2003). Activity of individual flies was displayed on actograms. For each individual fly, activity values for each $30 \mathrm{~min}$ bin in a $24 \mathrm{hr}$ time window were also averaged over successive days and normalized to that fly's total daily activity to yield average relative activities per bin. These values were further averaged over all flies in a given group and displayed as histograms of group average activity. Flies were considered rhythmic if their power (height above the 95\% confidence line of the major periodogram peak) was $>20$, the width of the major periodogram peak was $>3$, and their period within $4 \mathrm{hr}$ of the group mean (computed on flies that satisfied the first two criteria). Average temperatures differed by $<0.5^{\circ} \mathrm{C}$ between the light and dark portions of a cycle. Light intensity from cool white fluorescent lamps was measured at various positions inside the incubators with a Li-Cor (Lincoln, NE) Quantum Photometer. Up to three neutral density filters (Geste Scenique SA, France; 10\% transmittance checked with the photometer) were put on top of monitors to lower light intensity and down to $\sim 0.2$ lux for white light. Red Plexiglas filters were used to cut out all wavelengths $<600 \mathrm{~nm}$ (and $>700 \mathrm{~nm}$ ). Such a filter reduced intensity by a factor of five.

All experiments reported here were performed on virgin females, because they survive longer than males in the activity monitors. To evaluate entrainment efficiency, they were always first exposed to a light/dark (LD) schedule shifted 8-12 hr from normal laboratory schedule. Zeitgeber time (ZT) 0 is light-ON, and ZT 12 is light-OFF. After $6-7 \mathrm{~d}$ of entrainment, they were usually kept in constant conditions: LL for an additional $7 \mathrm{~d}$ and then in constant darkness (DD) for another 7-10 d. Circadian time (CT) 0 is $12 \mathrm{hr}$ after the last light-OFF (in DD). LLinduced arrhythmia for a given genotype was distinguished from illhealth effects by requiring an improved rhythmicity in the subsequent DD. The first $24 \mathrm{hr}$ in constant conditions were always excluded from period analyses.

In some experiments, the first schedule was shifted by an $8 \mathrm{hr}$ delay of light-OFF on the seventh full day to obtain a second LD schedule, which was maintained for at least $5 \mathrm{~d}$. The morning and evening activity peaks were determined for each fly and each day, using the filtered output of the data analysis package (Hamblen et al., 1986). The mean position (with its SE) of the two peaks for a given genotype was thus computed for each day and plotted over the course of the entire experiment (see Fig. 6).

Histology and imaging. Adult CNSs were dissected as described previously (Malpel et al., 2002), except for anti-CRY labeling, in which dissections were performed under red light, and fixation was done in the dark. 
Dilutions for the primary antibodies were as follows: mouse antichaoptin mAb24B10 (Fujita et al., 1982) and anti-GLASS mAb9B2.1 (Ellis et al., 1993) monoclonal antibodies, 1/100; rabbit anti-PER serum (Stanewsky et al., 1997) adsorbed against adult per $^{0}$ acetone powder, 1/15000; and guinea-pig anti-PDF antiserum (Neosystem Laboratoire, Strasbourg, France), 1/200. The rabbit anti-CRY antiserum was also raised by Neosystem Laboratoire against a peptide in the $\mathrm{N}$-terminal half of Drosophila CRY (amino acids 176-193). The serum was purified by affinity chromatography on the peptide (Q-BIOgene). Purified serum was used at 1/100 dilution. Secondary antibodies were Alexa594- and Alexa488-conjugated goat antibodies to rabbit IgG (1/5000 and 1/2000 dilutions, respectively), Alexa488-conjugated goat antibodies to guineapig IgG, and Alexa594-conjugated goat antibodies to mouse IgG, 1/1000 dilution (Molecular Probes, Eugene, OR). Images were made from an epifluorescence microscope (Axioplan2; Zeiss, Thornwood, NY) with a cooled digital camera (SPOT2; Diagnostic Instruments, Sterling Heights, $\mathrm{MI}$ ). Confocal imaging was performed on a Leica (Nussloch, Germany) SP2 confocal microscope.

\section{Results}

CRY protein accumulates in clock neurons of dark-reared larvae but is absent in the DN2s

cry gene expression in the larval brain was studied with a cry-gal4 insertion (cry-gal4-39), selected for driving high expression of a UAS- $g f p$ reporter (see Materials and Methods). As previously suggested by in situ hybridization (Emery et al., 2000b), cry was expressed in the larval LNs, which are the precursors to the small $\mathrm{LN}_{\mathrm{v}} \mathrm{s}$ (Fig. $1 A-F$ ). In the dorsal brain, cry expression was observed in the two larval DN1s but not in the DN2s. The selective absence of larval DN2 labeling was observed with two additional independent insertions of the same cry-gal4 construct (data not shown) and thus is probably not an artifact produced by positional effects on transgene expression.

To obtain direct evidence as to the expression pattern of the CRY protein, an anti-peptide antiserum was developed that worked on brain whole mounts (see Materials and Methods). It labeled the cell bodies of the LNs and DN1s, but not the DN2s, in wild-type larvae that were kept in permanent darkness (Fig. $1 H, K, L)$. Staining levels were weak, however, as can be qualitatively appreciated from the comparison with CRYoverexpressing larvae raised under the same conditions (Fig. 1, compare $H$ and $I)$. Exposure of the latter larvae to intense $(>100$ lux) white light for 1-2 hr completely abolished labeling (M.P., A.K., and F.R., unpublished data), consistent with the rapid lightinduced degradation of CRY reported in head extracts (Emery et al., 1998) or transfected cells (Lin et al., 2001). In confocal microscopy, anti-CRY labeling of axonal and dendritic processes was clearly observed in the larval LNs (Fig. $1 K, L$ ). The labeling of neuritic endings relative to cell bodies was quite similar for CRY (Fig. $1 H, I, L)$ and GFP (Fig. $1 G, J$ ).

\section{Forced cry expression in the larval DN2s brings their PER oscillations into phase with the other clock neurons}

The larval DN2s characteristically express PER in antiphase to the LNs and the DN1s (Kaneko et al., 1997) (Fig. 1A-F). We wondered whether this phase difference might be related to the absence of CRY in the DN2s. Overexpression of CRY protein was thus driven in all larval clock neurons, including the DN2s, with tim-gal4 (as documented in Fig. 1I). The controls had CRY overexpression restricted to either the LNs, using $p d f$-gal4 as a driver, or to the LNs and DN1s, with cry-gal4. No change was detected in the expression pattern of PER in either type of control (Fig. $2 A-D$, compare with Fig. $1 B, E$ ). In contrast, cry overexpression driven with tim-gal4 resulted in a complete phase reversal of PER

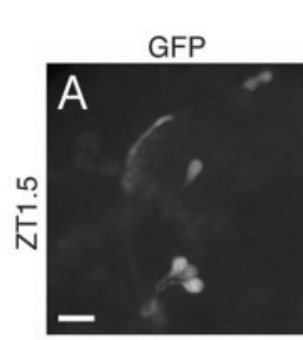

cry-gal4 UAS-gfp
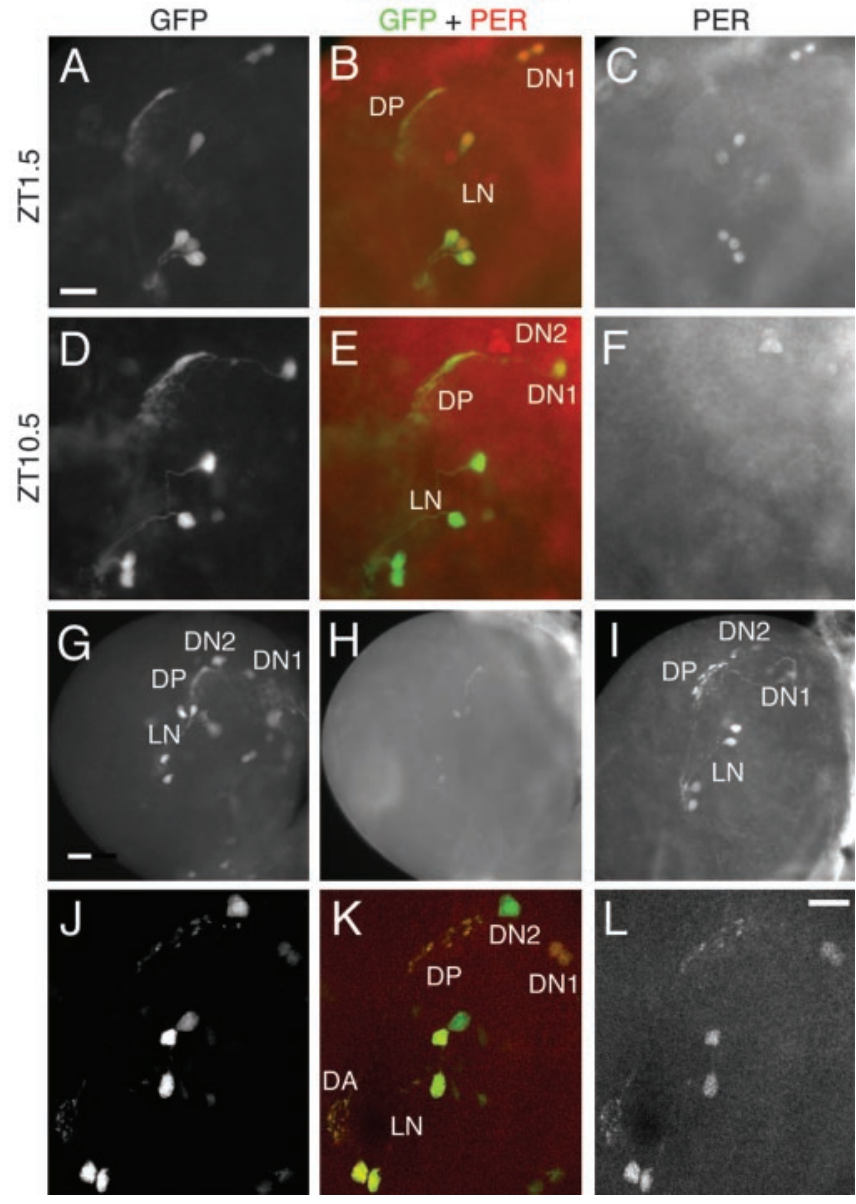

Figure 1. Expression of a cry reporter line (top panel) and the CRY protein (bottom panel) in the larval brain. $A-F$, PER labeling of third-instar cry-gal4 UAS-gfp larval brains at ZT1.5 ( $A-C$ or ZT10.5 (D-F). A, D, GFP staining. C, F, Anti-PER. B, E, Double labeling with GFP in green and PER in red. PER is expressed in the LNs and DN1s at ZT1.5 $(B, C$ and in the DN2s at ZT10.5 $(E, F)$. As described previously (Kaneko et al., 1997), there is a fifth larval LN associated to the four PDF-expressing ones, with a similar phase of PER expression in all five cells. No cry-gal4-driven GFP expression was ever detected in either the DN2s or the fifth LN. $G-L$, CRY labeling of tim-gal4/UAS-gfp $(G, H, J-L)$ or tim-gal4/UAS-cry (I) brains from third-instar larvae reared in the dark. In tim-gal4/UAS-gfp, the two DN1s and four LNs are double labeled (GFP, $G, J$, and green in $K ;(R Y, H, L$, and red in $K)$, but the two DN2s express only GFP. Wild-type (RY levels are very low compared with CRY levels achieved by tim-gal4-driven expression from a UAS-cry construct (compare $H$ and I). The dendritic arborization and dorsal projection of the LNs are more clearly visible in the confocal images in J-L.DP, Dorsal projection of the LNs; DA, dendritic arborization of the LNs. Scale bars, $20 \mu \mathrm{m}$.

cycling in the DN2s without any appreciable effect on the LNs or DN1s (Fig. 2E-G).

\section{The cry gene is expressed in most if not all clock neuronal} groups of the adult brain

In the adult brain, expression of a cry-gal4 transgene was clearly reported in the small $\mathrm{LN}_{\mathrm{v}} \mathrm{s}\left(\mathrm{s}-\mathrm{LN}_{\mathrm{v}} \mathrm{s}\right)$ and large $\mathrm{LN}_{\mathrm{v}} \mathrm{s}\left(1-\mathrm{LN}_{\mathrm{v}} \mathrm{s}\right)$ and was suggested in the $\mathrm{LN}_{\mathrm{d}} \mathrm{s}$ (Emery et al., 2000b). Using PER colabeling, we demonstrate here that the same construct is indeed expressed in all of the $\mathrm{LN}_{\mathrm{d}} \mathrm{s}$ (Fig. $\left.3 A-C\right)$. In addition, there was clear PER and cry-gal4 coexpression in the two DN2s (conversely to the larval ones), in most of the DN1s, and in $\sim 25 \%$ of the DN3s (Fig. 3D-F). All of these groups were observed with two additional cry-gal4 insertions (data not shown). Anti-CRY antibodies labeled no adult clock neurons at ZT8 (in $n=10$ hemi- 

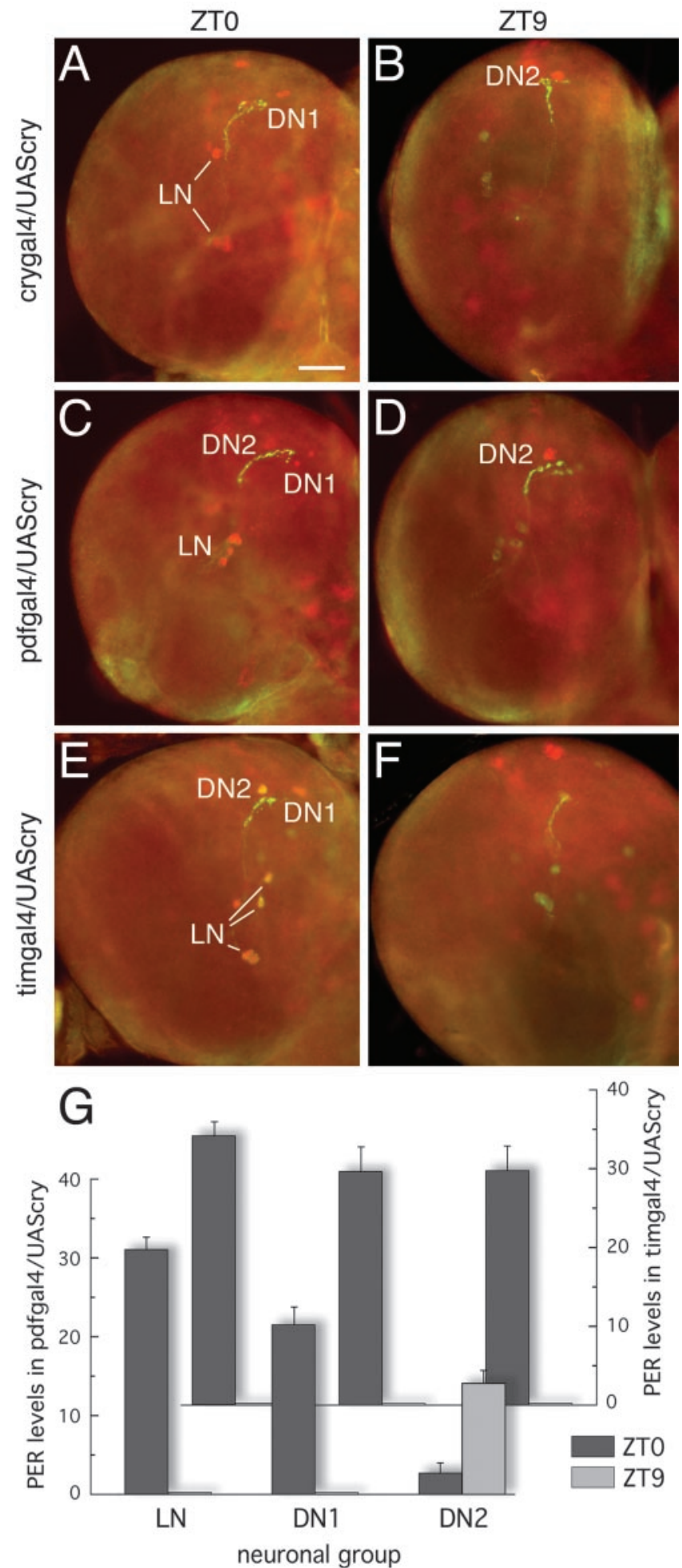

Figure 2. PER cycling in larval brain neurons overexpressing cry. A-F, cry-gal4/UAS-cry $(A$, $B)$, pdf-gal4/UAS-cry $(C, D)$, and tim-gal4/UAS-cry $(E, F)$ third instar larval brains labeled at ZTO-1 $(A, C, E)$ or ZT9-10 (B, D, F) with anti-PER antibodies (red). The cry-gal4/UAS-cry and pdf-gal//UAS-cry brains were double labeled with anti-PDF antibodies ( $A-D$, green). The DN2s always appear very close to the horizontal end-segment of the PDF-labeled dorsal projection from the LNs. The GFP reporter in panels $E$ and $F$ (green) allows even surer identification of the DN2s, but that was possible only with the tim-gal4 driver. Note that because of competition, the presence of the UAS-gfp transgene in these larvae might lower expression from UAS-cry, and thus strengthens the comparison with the other two genotypes. Weakly labeled DN2s could sometimes be seen at ZTO in cry-gal4/UAS-cry and pdf-gal4/UAS-cry brains (as illustrated in C). Similar results were obtained in at least two independent experiments for each genotype, spheres), although samples were systematically observed in confocal microscopy with tim-gal4-driven GFP to localize clock cells. Only very faint labeling was observed in only one s- $\mathrm{LN}_{\mathrm{v}}$ in a single brain at ZT23 $(n=9)$. However, an average of $1.1 \pm 0.1(n=10)$ $s-\mathrm{LN}_{\mathrm{v}}$ was labeled at CT6 (i.e., after $18 \mathrm{hr}$ in the dark), and all four s- $\mathrm{LN}_{\mathrm{v}} \mathrm{s}$ were systematically labeled after $3 \mathrm{~d}$ in $\mathrm{DD}(n=10$; data not shown) (Fig. $3 G-I$ ). These results are consistent with a very long half-life for CRY in the $\mathrm{s}-\mathrm{LN}_{\mathrm{v}} \mathrm{s}$ in the dark. Under prolonged dark exposure, at least some neurons in each one of the l- $\mathrm{LN}_{\mathrm{v}}$, $\mathrm{LN}_{\mathrm{d}}$, and DN1 groups were also labeled but none of the DN2s and DN3s (Fig. 3G-L) (data not shown). When flies were reared in DD throughout, CRY immunoreactivity was also found in the dorsal projections of the $s-\mathrm{LN}_{\mathrm{v}} \mathrm{s}$ (Fig. $3 J-L$ ), just as it was in the larval LNs (Fig. $1 K, L$ ). In most cells, both nucleus and cytoplasm were similarly labeled. No labeling was observed in $c r y^{b}$ mutant brains reared in DD (data not shown).

\section{Adult DN1s express the photoreceptor differentiation factor GLASS}

The circadian clock of $g l^{60 J} c r y^{b}$ double mutants has been reported to be completely blind. Surprisingly, the $g^{l^{60 J}}$ mutation removes not only the visual system but also a subset of PER-expressing DN1 neurons (Helfrich-Förster et al., 2001). Double-labeling experiments showed that the GLASS and PER proteins are coexpressed in the DN1 neurons of wild-type flies (Fig. 4A,D). Colocalization was confirmed by confocal microscopy (data not shown). The number of colabeled cells approximately matched the number of DN1s that are missing in $g l^{60 J}$ brains (Fig. $4 B, E$; quantified in $N$ ). The DN2s were not affected in the mutant. The other two remaining cells $\left(\mathrm{DN}^{*}\right)$ probably correspond to the two larval DN1s (Kaneko et al., 1997), which did not express GLASS and were present in $g l^{60 J}$ larvae as inferred from anti-PER labeling (data not shown). GLASS expression in the adult therefore distinguishes between the DN1s that were already present in larvae and those that appear during metamorphosis. Two additional cell groups, one comprising 40-50 cells and the other 7-8 cells, strongly expressed GLASS in the adult wild-type brain (Fig. $4 G, K)$. None of these extra cells coexpressed PER, although the smaller group was quite close to the $\mathrm{LN}_{\mathrm{d}} \mathrm{s}$, with which it was often interspersed (Fig. 4K).

GMR-hid flies are devoid of any of the known glassdependent, rhodopsin-based photoreceptors, including the HB fiber (see Materials and Methods). However, staining of GMRhid brains with an anti-PER antiserum showed the normal complement of PER-expressing neurons, including the DN1s (Fig. $4 C, F, I, M, N)$. We did not observe PER or cry-gal4 expression in any of the GLASS-positive cells described in larval brains (Hassan et al., 2000; Malpel et al., 2004). These larval cells formed one small cluster ( $8.8 \pm 0.3$ cells; $n=14$ hemispheres) and two large clusters (ventral, 30-50 cells; dorsal, $18.9 \pm 0.3$ cells; data not shown) similar in size and location to those found in the adult.

\section{Absence of the adult DN1s correlates with a lower light sensitivity of the circadian clock}

Because the adult DN1 neurons are the only clock cells absent in $g l^{60 \mathrm{O}}$ brains and present in GMR-hid brains, we decided to test

$\leftarrow$

both with and without anti-PDF colabeling (or GFP coexpression). PER immunoreactivity was quantified in the LNs, DN1s, and DN2s (G) for pdf-gal4/UAS-cry and tim-gal4/UAS-cry larval brains dissected at either ZTO or ZT9. The front and back histograms correspond to pdf-gal4/ UAS-cry and tim-gal4/UAS-cry values, respectively. Error bars indicate SEMs. At least 19 hemispheres were scored per sample, except for tim-gal4/UAS-cry at ZT9 $(n=12)$. Scale bar, $40 \mu \mathrm{m}$. 
their putative role in circadian photoreception by comparing the light sensitivity of the two lines. Two different light regimes were used, LD entrainment and constant light. The norpA $A^{p 24}$ mutant, which abolishes rhodopsin-based photoreception (the norpA gene encodes a phospholipase component of the phototransduction cascade) and has normal numbers of DN1s, was also included in most comparisons. At high intensity, the wild-type, GMR-hid, norp $A^{p 24}$, and $g l^{60 J}$ flies rapidly resynchronized after the LD schedule was delayed by $8 \mathrm{hr}$ (Fig. 5). At a 1000-fold lower intensity, all mutants synchronized to the new schedule slower than the wildtype, but $g l^{60 J}$ flies synchronized much less efficiently than GMR-hid or norp $A^{p 24}$ flies (Figs. 5, 6). As reported previously (Helfrich-Förster et al., 2001), the strongest $g l^{60 J}$ defect was in the resetting of the morning activity peak (Fig. 6). In contrast, the morning peak of GMR-hid flies synchronized as rapidly as the evening one, and both peaks synchronized faster than in the $g l^{60 J}$ flies. Entrained GMR-hid and $g l^{60 J}$ flies also had very different activity patterns (Fig. 6).

Constant light results in intensitydependent arrhythmia in wild-type Drosophila (Konopka et al., 1989), whereas cry $^{b}$ mutants stay strongly rhythmic (Emery et al., 2000a) with a lengthened period (Helfrich-Förster et al., 2001). We observed that $g l^{60 J}$ flies were much less severely affected than wild-type and rather comparable with $c r y^{b}$ (Table 1). Their period was significantly increased, even at the lowest light intensity tested. In contrast, both GMR-hid and norp $A^{p 24}$ flies appeared at least as sensitive to LL as the wild type (Table 1). Together, these results show that the clock is more sensitive to light in GMR-hid and norp $A^{p 24}$ than in $g l^{60 J}$ flies and strongly suggest that the adult-specific DN1s contribute to circadian photoreception.

Light responses of visual systemdepleted flies depend on cryptochrome activation

Because cry is expressed in the DN1s, we tested whether the difference in light sensitivity between GMR-hid and $g l^{60 J}$ flies depends on cryptochrome. Like $g l^{60 J} \mathrm{cry}^{b}$ flies, the GMR-hid; cry strain did not entrain to LD cycles of even the highest amplitude, although the activity pattern of the two strains differed markedly (Fig. 7). We also tested entrainment to red light/dark cycles. Under these conditions, CRY should not be activated at all (Suri et al., 1998; Ahmad et al., 2002). Wild-type flies synchronized to such cycles; however, neither GMR-hid nor $\mathrm{gl}^{60 J}$ flies did (data not shown), which is consistent with CRY
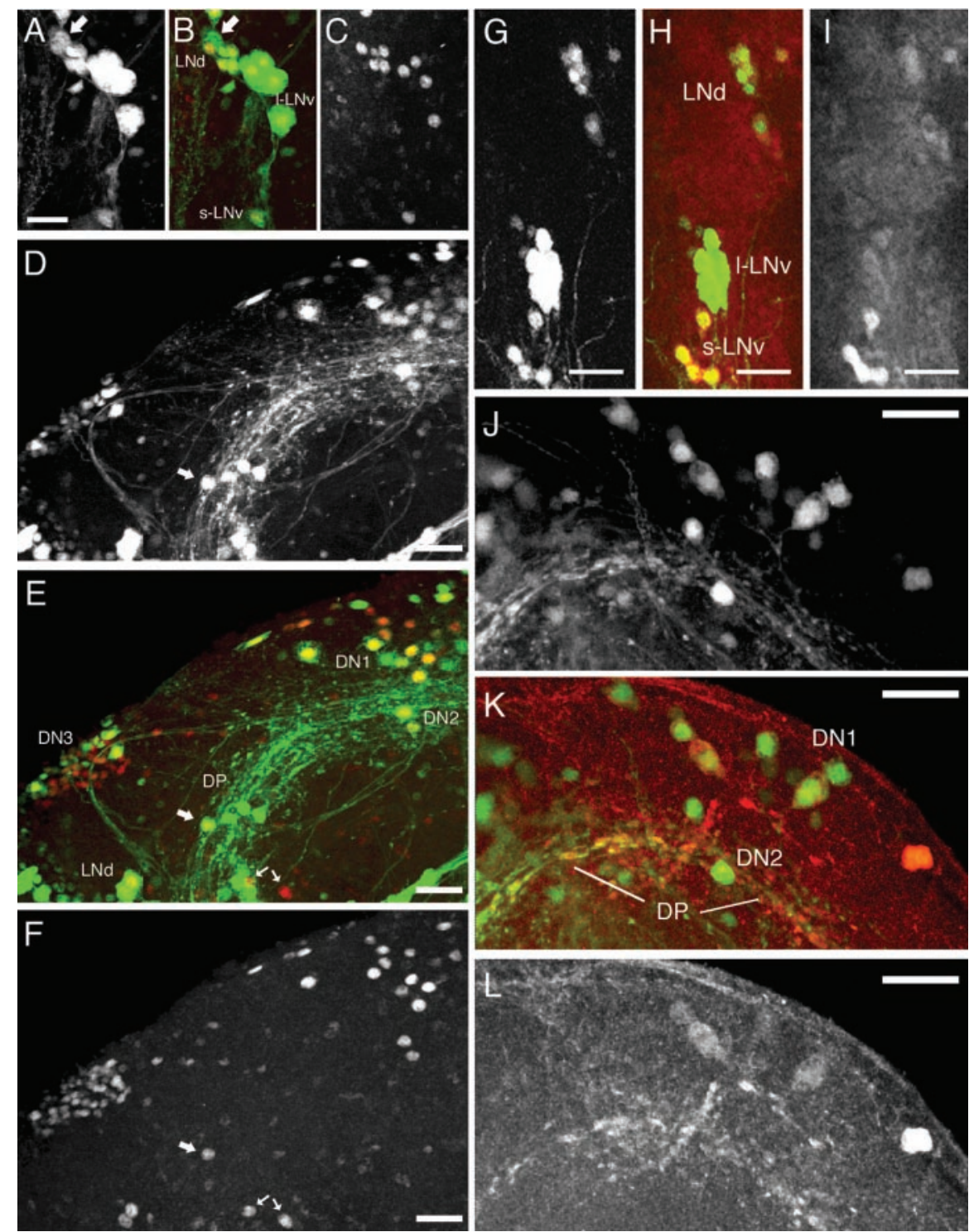

Figure 3. Expression of a cry reporter line and the CRY protein in the adult brain. $A-F$, Anti-PER labeling at ZT1-2 in a cry-gal4 UAS-gfp brain. A-C, Confocal projection of the region of I-LN $\mathrm{v} s$ and $\mathrm{LN}_{d} \mathrm{~s}$. $A, B$, Green, GFP; $B$, Red, C, PER. All $\mathrm{LN}_{v}$ s and $\mathrm{LN}_{\mathrm{d}} \mathrm{s}$ were always GFP positive. The arrow indicates a GFP-positive cell without PER expression, which was reproducibly observed within the $\mathrm{LN}_{\mathrm{d}}$ cluster. D-F, Confocal projection of the dorsal brain. $D, E$, Green, GFP; $E$, Red, F, PER. Most of the DN1s (11.4 \pm 0.5 of $15.2 \pm$ $0.6 ; n=5$ brain hemispheres) expressed GFP. In the DN3 group, comprising a total of 30 40 cells (Kaneko et al., 1997; Kaneko and Hall, 2000), the number of clearly GFP-positive cells was only $8.5 \pm 0.4(n=11)$. Two or three of these stood out with much stronger GFP expression and a much larger cell body. The DN2s lie very close to the dorsal projection (DP) of the s-LN $\mathrm{L}_{\mathrm{v}} \mathrm{s}$. The many additional PER-expressing cells are probably glial (Kaneko, 1998), except possibly for the three cells indicated by an arrow. As judged from their number and position, they could correspond to the novel cluster of three TIM-positive cells described by Kaneko and Hall (2000). One (thicker arrow) is GFP positive and displays an axon-like projection (data not shown). G-L, Anti-CRY labeling. $\mathrm{G}-\mathrm{I}$, Confocal projection of the region of the lateral neurons in a cry-gal4 UAS-gfp brain. G, H, Green, GFP; H, Red, I, CRY. After rearing in $L D$, adult flies were kept for $3 \mathrm{~d}$ in DD before dissection. All four $s-\mathrm{LN}_{v} s$ were always labeled with the anti-CRY antibodies, whereas only variable proportions of the $I-L_{v} s$ and $L_{d} s$ were labeled, usually more weakly than the $s-L N_{v} s$. J-L, Confocal projection of the dorsal brain of an adult tim-gal4/UAS-gfp brain. J, K, Green, GFP; K, Red, L, CRY. Flies were reared in constant darkness throughout. An average of $6.1 \pm 0.3 \mathrm{DN} 1 \mathrm{~s}$ were CRY positive ( $n=8$ brain hemispheres). Two of them were usually much more strongly labeled than the others, as shown here. Double labeling of wild-type brains with anti-CRY and anti-GLASS antibodies (data not shown) allowed us to identify them as larva-originating DN1s (Fig. 4). The dorsal projections of the s-LN $s$ were labeled in seven of eight brain hemispheres. Scale bars, $20 \mu \mathrm{m}$.

being the only pathway of entrainment by light for visual systemdepleted flies.

The $c r y^{b}$ mutation very efficiently suppresses the arrhythmia of wild-type flies in LL (Emery et al., 2000a) (Table 1). It did the same for all visual system-defective strains (e.g., $93 \pm 4 \%$ of 

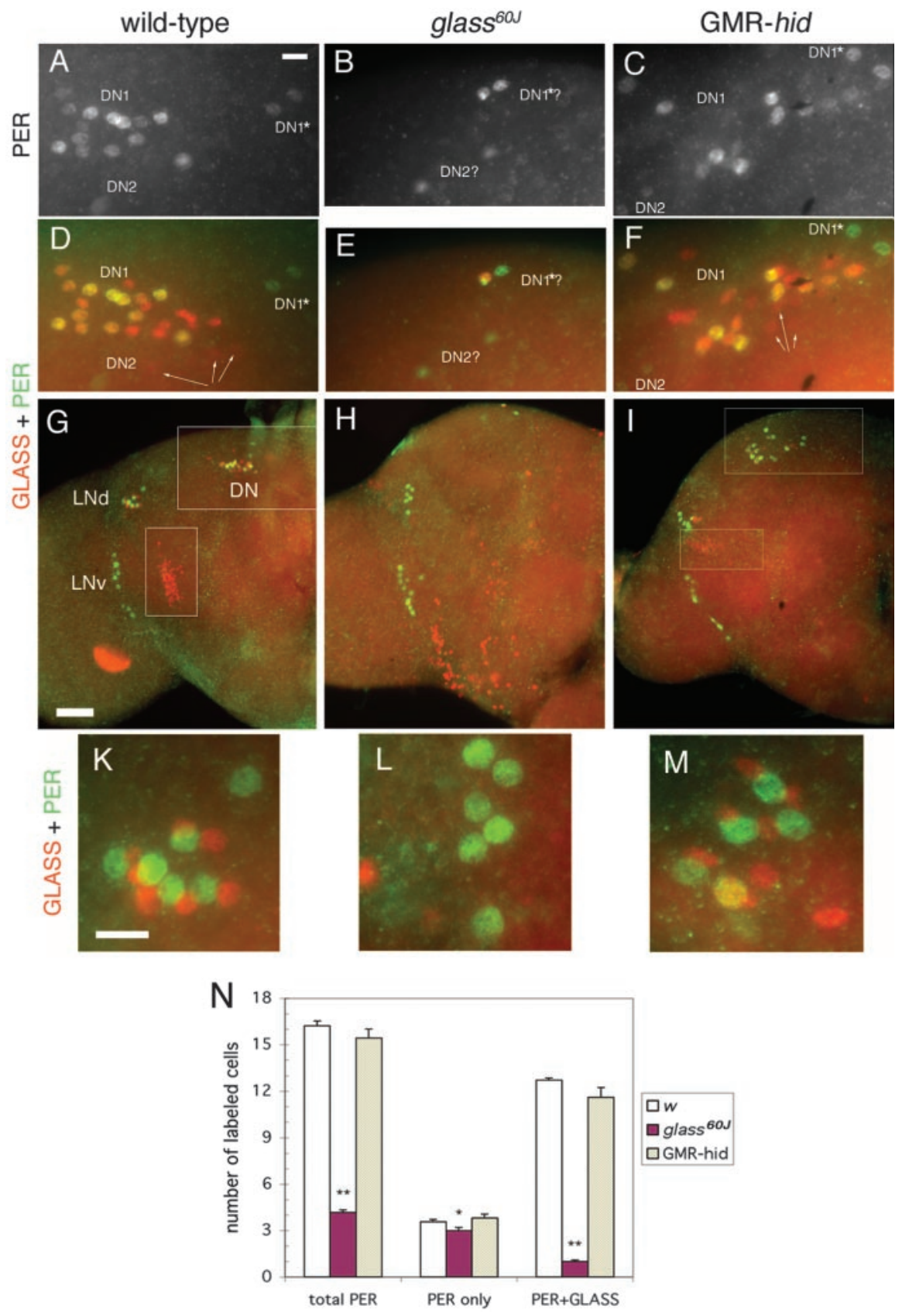

Figure 4. PER and GLASS expression in wild-type, $g l^{60 J}$, and GMR-hid adult brains. Labeling was done on brains collected at CTO. A-C, Anti-PER. D-M, Anti-PER (green) and anti-GLASS (red) colabeling. The two DN2s usually formed a close pair, more ventral and in a different plane of focus than the DN1s, as described previously (Kaneko et al., 1997) and as shown here $(A-F)$. However, cell identification was not always certain (hence the "?" in panels $B$ and $E$ ). The number of GLASS-positive cells in the wild-type dorsal clusters was $22.2 \pm 0.4(n=9)$. An additional five to six cells appeared weakly but very reproducibly GLASS positive in that region ( $D, F$, arrows). The $D N 1^{*} s$, which do not express GLASS, presumably correspond to the two larval DN1s. They were often in a more dorsal position than the other DN1s. The dorsal neurons are the only cells that coexpress PER and GLASS in adult brains. $G-$, Half-brains. $K-M$, Higher magnification of $\mathrm{LN}_{\mathrm{d}}$ regions. The largest GLASS-expressing cluster (variably positioned, depending on the orientations of the optic lobe and central brain on the slide) and the DNs are in a different frontal plane than the smallest GLASS-expressing cluster and the LNs. The thinly boxed areas in $G$ and / were thus taken from another differently focused picture. In $\mathrm{gl}^{60 J}$ brains, the anti-GLASS antibody labeled a completely different set of scattered and mostly ventral cells $(E$, $H, L)$. There was reproducibly a single colabeled cell $(E)$. From its very dorsal localization and its persistence in the mutant, we tentatively identify it as one of the two DN1*s (which do not express GLASS in the wild type; see D). No GLASS-expressing cell seemed to be missing in GMR-hid brains $(F, I, M)$. Quantification of PER-expressing dorsal neurons in control, $g l^{60 J}$, and GMR-hid adult brains is shown in N. Significant differences relative to control are indicated ( ${ }^{*} p<0.05 ;{ }^{* *} p<0.001$; Student's $t$ test; one-tailed, after testing for differences in variance). Because it was not always possible to distinguish between the three subgroups of dorsal neurons as easily as on the examples shown in $A-F$, all of these PER-expressing cells were systematically counted together. $g l^{60 J}$ mutant brains display less (PER only) neurons than control or GMR-hid flies, because one of the presumptive DN1*s is colabeled in the mutant but not in the other two strains. Scale bars: $A-F, K-M, 10 \mu \mathrm{m} ; G-I, 50 \mu \mathrm{m}$.
GMR-hid; $c r y^{b}$ flies were rhythmic in LL; $n=3$ experiments at 250-1000 lux). However, the period lengthening of $c r y^{b}$ flies in LL was suppressed in the $g^{60 J}$, GMR-hid, and norp $A^{p 24}$ backgrounds, even at the highest light intensity (Table 2 ). This period lengthening thus requires a functional visual system.

\section{PER oscillations in the DN1s require PDF-expressing $\mathrm{LN}_{\mathrm{v}} \mathrm{s}$}

Adult DN1 neurons display PER oscillations for at least $1 \mathrm{~d}$ in constant darkness in wild-type flies and in flies ablated for the PDF-expressing $\mathrm{LN}_{\mathrm{v}} \mathrm{s}$ (Blanchardon et al., 2001). Such PER oscillations have been proposed to play a role in the weak shortperiod rhythmicity found in mutant strains such as disco or $p d f^{01}$ (HelfrichFörster, 1998; Renn et al., 1999; Blanchardon et al., 2001). To test that hypothesis, we produced $p d f^{01} g l^{60 J}$ recombinants, which lack the adult DN1s, and compared them with DN1s-retaining GMR-hid; $p d f^{01}$ flies. Both of these strains had rhythms quite similar to those of the $p d f^{01}$ mutant (Table 3), suggesting that their rhythmicity does not involve the adult DN1s. Furthermore, oscillations of PER levels dampened rapidly in the DN1s of $p d f^{01}$ flies after transfer to DD (Fig. $8 B, D, E)$, whereas robust PER cycling persisted for at least $2.5 \mathrm{~d}$ in their $\mathrm{s}-\mathrm{LN}_{\mathrm{v}} \mathrm{s}$, 1-LN $\mathrm{L}_{\mathrm{v}} \mathrm{s}, \mathrm{LN}_{\mathrm{d}} \mathrm{s}$, and DN3s (data not shown). In wild-type flies, PER cycling persisted in all neuronal groups for at least $2 \mathrm{~d}$ in $\mathrm{DD}$ (Fig. 8A, $C, E$ ) (data not shown). We therefore conclude that the adult DN1 neurons play no part in the rhythmicity of the $p d f^{01}$ flies but rather are the oscillators that are most dependent on PDF expression in the $\mathrm{LN}_{\mathrm{v}} \mathrm{s}$.

\section{Discussion \\ Unexpected intercellular and intracellular distribution of CRY protein}

Use of a novel anti-CRY antiserum showed expression of CRY protein in most clock neurons, with the exception of the larval DN2s and the adult DN2s and DN3s. Lack of cry expression in the larval DN2s is supported by the absence of crygal4-driven labeling of these cells and by the remarkable reversal of the phase of their PER cycling when they are forced to express cry (see below). Conversely, crygal4 expression in adult DN2s and DN3s strongly suggests that our failure to detect the CRY protein in these neurons is because of its low levels. Indeed, recent in situ hybridization data showed cry expression in several groups of adult dorsal neurons, 

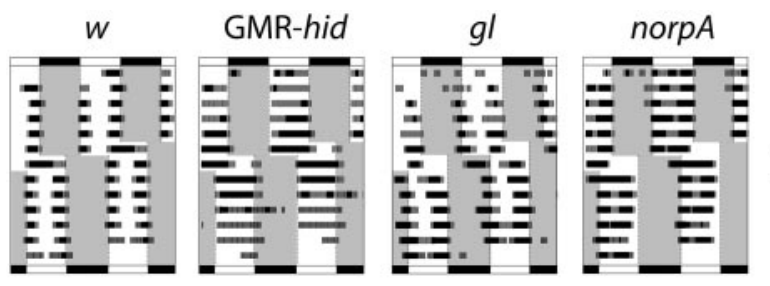

high
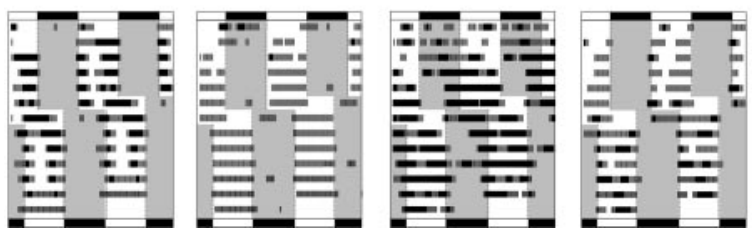

low

Figure 5. Actograms of individual control and visual system-defective flies submitted to a shift in LD schedule at either high- or low-light intensity. Light intensity was 80 -200 lux for the top panel and $0.08-0.2$ lux for the bottom panel. For the first half of the experiment, lights were on from 8:00 P.M. to 8:00 A.M., almost completely antiphase to the normal lab schedule that the flies had previously been exposed to. The LD schedule was then shifted by $8 \mathrm{hr}$ on the seventh day by delaying the light-OFF transition, as indicated by the broken lines. At low light intensity, the glass ${ }^{60 J}$ fly clearly took longer than the $w$, GMR-hid, and norp $A^{p 24}$ to entrain to both the first LD schedule and the subsequent $8 \mathrm{hr}$ shift. These actograms are representative of two to three independent experiments, each with at least 12 flies for each experimental condition.

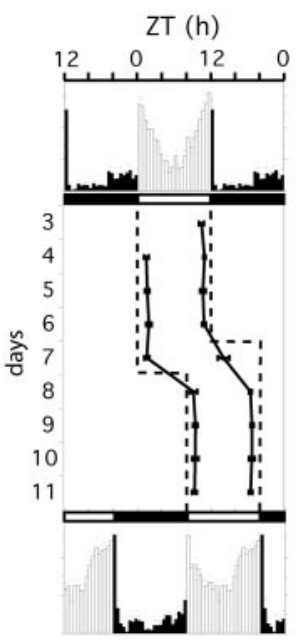

W

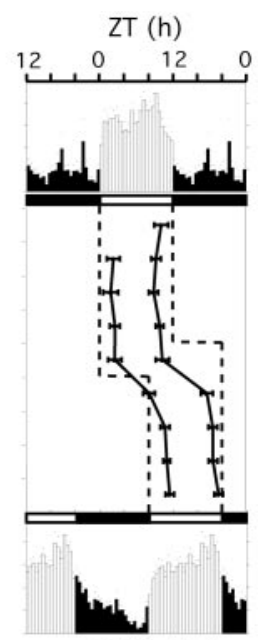

GMR-hid

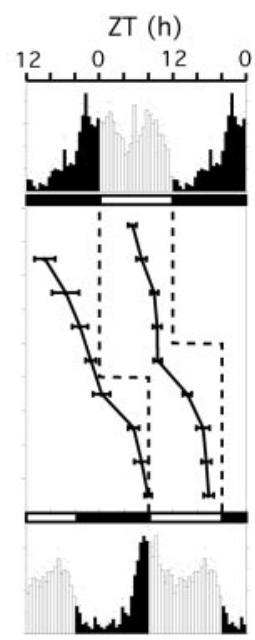

$g l^{60 J}$
Figure 6. Average daily activity profiles of groups of control, GMR-hid, and $g /{ }^{60 J}$ flies before and after a shift in LD schedule using a low ( $0.08-0.2$ lux) intensity of light. Activity profiles are averages for all flies of a given genotype $(n=12-25)$ over the last $2 \mathrm{~d}$ of the corresponding $L D$ schedule. The average morning and evening activity peaks were computed for each day as described in Materials and Methods. GMR-hid flies maintained almost constant activity throughout the light interval, sometimes precluding a clear determination of the activity peaks (especially the morning peak). Entrained $g^{60 J}$ flies significantly anticipated the light- $0 \mathrm{~N}$ transition in contrast to the GMR-hid or wild-type flies. This anticipation may also account for the apparent lack of acute response of the $g^{60 J}$ flies to light- $0 \mathrm{~N}$, again contrasting with the two other genotypes.

including the DN1s and DN3s (Zhao et al., 2003). Moreover, the adult DN2s are the only brain neurons in which PER cycling is completely abolished by the $c r y^{b}$ mutation (Helfrich-Förster et al., 2001), suggesting that CRY plays a role in these neurons.

Under LD conditions, CRY levels remain very low in all brain neurons, consistent with light-dependent CRY degradation (Emery et al., 1998; Lin et al., 2001). We could detect CRY reliably in wild-type adult brains only after prolonged dark exposure. In
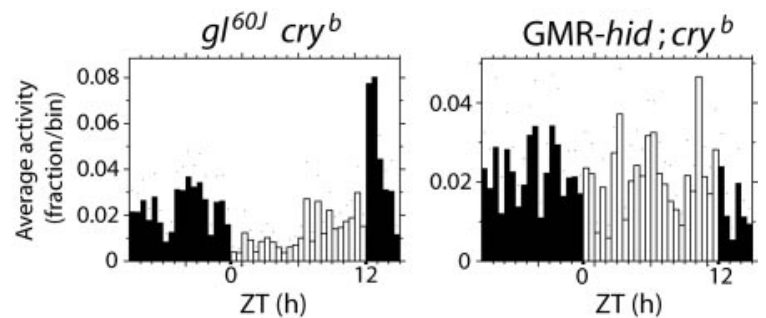

Figure 7. Average activity profiles of $g^{60 J} \mathrm{Cry}^{b}$ and GMR-hid; $c r y^{b}$ flies entrained with intense ( $\sim 500$ lux) white light. Note that GMR-hid; cry $^{b}$ behaved differently from $\mathrm{gl}^{60 J} \mathrm{Cry}^{b}$ flies, in that their activity was neither suppressed during the day nor transiently induced by the light-OFF transition. These profiles were obtained by averaging the activity of 16 flies of each genotype during the fourth full day of entrainment.

Table 2. Effect of visual mutations on the period of rhythmic cry ${ }^{b}$ flies in constant light

\begin{tabular}{|c|c|c|c|c|}
\hline Genotype & $500 \operatorname{lux}^{a}$ & $50 \operatorname{lux}$ & $5 \operatorname{lux}$ & $0.5 \operatorname{lux}$ \\
\hline$c r y^{b} s s$ & $25.4 \pm 0.1$ & $25.0 \pm 0.5$ & $24.0 \pm 0.3$ & $23.4 \pm 0.3$ \\
\hline$w ; c^{b} y^{b} s s$ & $25.3 \pm 0.1$ & ND & ND & ND \\
\hline w; GMR-hid; cry ss & $23.6 \pm 0.1$ & $23.3 \pm 0.1$ & $23.4 \pm 0.1$ & ND \\
\hline$w ; g^{60 J} \mathrm{cry}^{b}$ & $23.8 \pm 0.1$ & $23.7 \pm 0.2$ & $23.5 \pm 0.1$ & $23.7 \pm 0.1$ \\
\hline w norp $A^{p 24} ; c^{b}{ }^{b}$ ss & $23.1 \pm 0.1$ & ND & ND & ND \\
\hline
\end{tabular}

Periods were obtained from periodogram analysis for flies kept in $L \mathrm{~L}$ for at least 7 d. Values shown are means \pm SEM (in hours) of two to five independent experiments, each with 8-16 rhythmic flies for each genotype and light intensity. Values for $c r y{ }^{b}$ ss flies are repeated from Table 1 for easier comparison. ND, Not determined.

a Light intensity experienced by individual flies varied by less than a factor of two around the values shown.

Table 3. Role of the adult DN1s in generating activity rhythms

\begin{tabular}{|c|c|c|c|}
\hline Genotype & $\begin{array}{l}\text { Percentage of } \\
\text { rhythmic flies }(n)^{a}\end{array}$ & Period \pm SEM (hr) & Power \pm SEM \\
\hline$y w$ & $100 \%(30)$ & $23.9 \pm 0.1$ & $100 \pm 4$ \\
\hline$y w ; p d f^{01}$ & $69 \%(42)^{b}$ & $22.4 \pm 0.2$ & $55 \pm 4$ \\
\hline w & $93 \%$ (29) & $23.7 \pm 0.1$ & $104 \pm 6$ \\
\hline$w ; p d f^{01}$ & $64 \%(47)$ & $22.7 \pm 0.3$ & $49 \pm 4$ \\
\hline w; GMR-hid & $100 \%(15)$ & $24.3 \pm 0.3$ & $87 \pm 6$ \\
\hline w; GMR-hid; pdf ${ }^{01}$ & $63 \%(30)$ & $23.1 \pm 0.1$ & $57 \pm 6$ \\
\hline$w ;\left.g\right|^{60 J}$ & $84 \%$ (32) & $23.8 \pm 0.1$ & $83 \pm 6$ \\
\hline$w ; g^{60 J} p d f^{01}$ & $69 \%(45)$ & $23.0 \pm 0.2$ & $64 \pm 6$ \\
\hline
\end{tabular}

${ }^{a}$ Virgin female flies were entrained in LD for $3-4 d$ and then kept in DD for $6 \mathrm{~d}$. $n$, Total number of flies alive at the end of the experiment. ${ }^{b}$ Fly strains lacking PDF expression were previously reported to be $2-63 \%$ rhythmic when males were assessed over $9 \mathrm{~d}$ of DD (Renn et al., 1999). Because rhythmicity was also reported to decrease with time in DD and because females tend to display more robust rhythmicity than males, these figures appear consistent with ours.

addition, CRY protein accumulates to very different levels between neuronal groups, levels which do not generally correlate with the strength of cry gene expression as inferred from cry-gal4 (Emery et al., 2000b; Zhao et al., 2003; this study) and in situ hybridization data (Zhao et al., 2003). This reveals an unexpected level of cell-specific post-transcriptional control of CRY expression. Only the cells that express CRY already in larvae, four $\mathrm{s}-\mathrm{LN}_{\mathrm{v}} \mathrm{s}$ and two of the DN1s, were reproducibly strongly labeled. This may in part reflect longer CRY accumulation. However, stronger labeling of the s- $\mathrm{LN}_{\mathrm{v}} \mathrm{s}$ also occurred when flies were transferred to constant darkness only after their eclosion as adults, suggesting higher stability of the CRY protein in these cells. The $c r y^{b}$ mutation differentially affects the amplitude of LD PER cycling in the different neuronal groups of the adult brain (Helfrich-Förster et al., 2001). Variations of CRY levels among brain neurons may therefore reflect functional differences.

Intracellularly, CRY clearly accumulates in both the nucleus and cytoplasm, including, at least in s- $\mathrm{LN}_{\mathrm{v}} \mathrm{s}$, axonal and dendritic projections. The presence of CRY in neurites provides another 

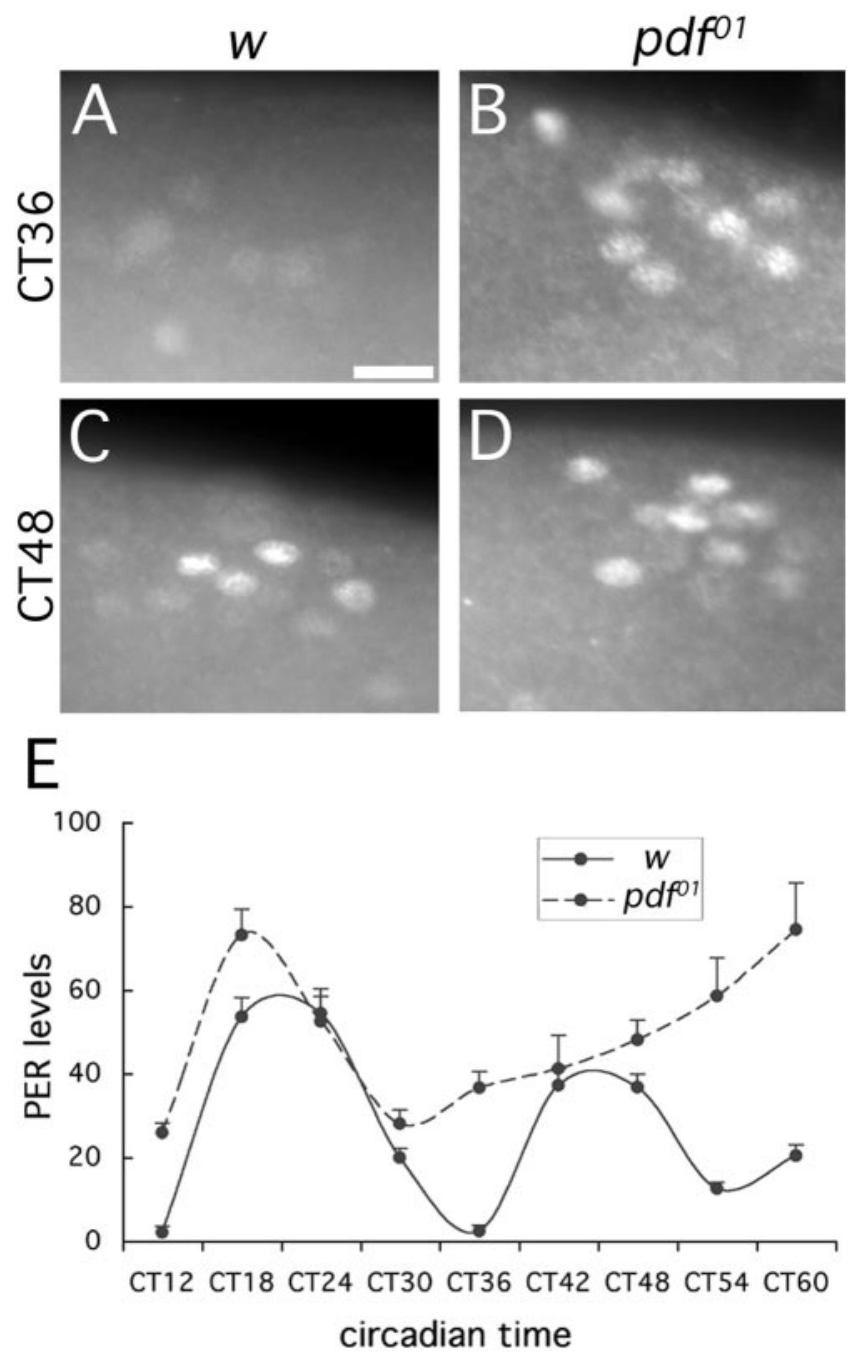

Figure 8. PER cycling in the DN1s of wild-type and $p d f{ }^{01}$ adult brains. $A-D$, Dorsal region from wild-type $(A, C)$ and $p d f^{01}(B, D)$ adult brains stained with anti-PER antibodies at either CT36 $(A, B)$ or $C T 48(C, D)$. Similar results were obtained in two independent experiments for each genotype, one of which extended to CT74 (i.e., $>3$ full days of $D D$ after the last dark cycle of the entraining LD schedule). $E$, Quantization of PER levels in the DN1s from the experiment illustrated in $A-D$. PER levels are mean values over an average of 16 brain hemispheres per sample. Error bars indicate SEMs.

potential level of CRY function and regulation and suggests that it may interact with proteins other than TIM.

\section{Functional significance of the lack of CRY expression in larval DN2s}

The developmental change in cry-gal4 expression in the DN2s correlates with the inversion of PER and TIM oscillations, which occurs in these cells during metamorphosis, bringing them into phase with the other brain clock cells (Kaneko et al., 1997, 2000). Driving CRY expression with a tim-gal4 driver conferred a normal phase to PER cycling in the larval DN2s without affecting it in other neurons. In contrast, when CRY overexpression was not extended to the DN2s, it did not alter PER cycling in the central brain, consistent with CRY acting autonomously in the larval DN2s to set their phase. Our results therefore indicate that CRY is sufficient to induce light-dependent degradation of TIM in brain neurons and to synchronize them accordingly. What then sets the (opposite) phase of wild-type larval DN2s? Their accumulation of TIM in the presence of light already suggested that they were intrinsically blind and thus had to rely on signals coming from light-sensitive neurons, which may include the PDF-expressing LNs that project nearby (Kaneko et al., 1997).

In contrast, the phase of PER and TIM cycling in the other larval brain neurons does not depend on CRY (Kaneko et al., 2000; Ivanchenko et al., 2001). This is consistent with these clock cells receiving inputs from the visual system, as shown for the LNs (Malpel et al., 2002).

\section{Cryptochrome-based photoreceptive capabilities of the adult DN1s}

Our results indicate that a structure present in GMR-hid flies and absent in $g l^{60 J}$ flies contributes in a CRY-dependent manner to the entrainment of the adult behavioral clock by LD cycles and to its sensitivity to constant light. The absence of the adult DN1s in the $g l^{60 J}$ mutant and the expression of CRY in these cells strongly point to their involvement in this light input pathway. A role for clock cells other than the $\mathrm{LN}_{\mathrm{v}} \mathrm{s}$ in CRY-dependent light responses was already suggested by targeted rescue experiments (Emery et al., 2000b). In addition to the $\mathrm{LN}_{\mathrm{v}} \mathrm{s}$ and the adult DN1s, the CRY expression profile suggests additional contributions from at least the $\mathrm{LN}_{\mathrm{d}} \mathrm{s}$ and the larva-originating DN1s, but their role in circadian photoreception remains unknown.

CRY-mediated photoreception is required for the entrainment of flies devoid of any visual structure, because GMR-hid; $c r y^{b}$ flies are circadianly blind. Conversely, the latter result also shows that CRY-independent pathways are confined to the visual system. Thus, the reported entrainment of norpA; $c r y^{b}$ flies by LD cycles (Stanewsky et al., 1998) must still rely on the visual system via either a residual function of the mutant no-receptor-potential A (NORPA) protein or a distinct phospholipase isozyme (Shortridge et al., 1991; Riesgo-Escovar et al., 1995).

Expression of GLASS in the adult DN1s indicates that the absence of these cells in the $\mathrm{gl}^{60 J}$ mutant may be a cellautonomous effect of the mutation. Indeed, the larval DN1s, which do not express GLASS, are not eliminated by the $g l^{60 J} \mathrm{mu}-$ tation and persist in $g l^{60 J}$ adult brains. In the adult brain, GLASS and CRY coexpression occurs only in adult DN1s, encouraging further speculation as to a specific photoreceptive role for these cells. GLASS, which is required for the differentiation of rhodopsin-expressing photoreceptors, may control the expression in the adult DN1s of a rhodopsin-based CRY-independent photoreceptive pathway. However, the circadian blindness of GMR-hid; $c r y^{b}$ flies appears to exclude a CRY-independent contribution of nonvisual system cells to circadian photoreception.

Why are the DN1s preserved in GMR-hid brains? GLASSexpressing cells similarly survive in GMR-hid larval brains, whereas the larval photoreceptors disappear (Hassan et al., 2000; Malpel et al., 2004). In contrast, all GLASS-expressing clusters are absent from adult $g l^{60 J}$ mutant brains. The latter still express a GLASS-related epitope in ectopic locations, which may be attributable to the aberrant expression of either a truncated GLASS protein or a glass-related gene in $g l^{60 J}$ mutants. Such widespread perturbation of gene expression in $g l^{60 J}$ brains may be relevant to the surprising noncircadian light responses of $\mathrm{gl}^{60 J} \mathrm{cry}^{b}$ (HelfrichFörster et al., 2001) but not GMR-hid; $c r y^{b}$ flies. Alternatively, a cry-independent function of the DN1s may prevent such noncircadian (or masking) effects to occur in GMR-hid; $c r y^{b}$ flies.

How do the DN1s contribute to circadian activity rhythms? In Drosophila, an intensity-dependent increase of period was reported in LL between 0.1 and 10 lux, above which most individuals became arrhythmic (Konopka et al., 1989). Although no 
consistent trend in period lengthening with intensity was seen in our two control strains, arrhythmia did appear at approximately the expected intensity (between 0.5 and 5 lux). $c r y^{b}$ mutants remain strongly rhythmic in LL and display longer periods than in DD (Helfrich-Förster et al., 2001; this study). Helfrich-Förster et al. (2001) found that most $g l^{60 J}$ flies were arrhythmic in LL, whereas the rest displayed long periods. We found a majority of rhythmic $g l^{60 J}$ flies in LL with long period rhythms. The rhythmicity of $g l^{60 J}$ flies in LL contrasts with the strong arrhymicity of blind GMR-hid and norp $A^{p 24}$ flies. Our data suggest that the absence of CRY-expressing adult DN1s may contribute to the persisting rhythms of both $c r y^{b}$ and $g l^{60 J}$ mutants in LL and to their period lengthening. However, the wild-type period of the double mutants $g l^{60 J} c r y^{b}$, GMR-hid; $c r y^{b}$, and norp $A^{p 24}$; $c r y^{b}$ in LL indicates that period lengthening in $c r y^{b}$ flies requires a functional visual system. Interactions between the visual system and CRYexpressing clock cells may modulate the electrical activity of the LNs, which was recently proposed to be an essential element of their molecular clock (Nitabach et al., 2002). The reported projections from the DN1s toward the $s-\mathrm{LN}_{\mathrm{v}} \mathrm{s}$, and from the $\mathrm{LN}_{\mathrm{d}} \mathrm{s}$ to the DN1s and DN2s (Kaneko and Hall, 2000), indeed suggest that all of these PER-expressing cells form a functional network, which may include the visual system.

The strong rhythmicity of $g l^{60 J}$ flies in constant darkness argues against a major role of the adult DN1s in generating behavioral rhythms. PER coding sequences under glass control restored some long-period rhythmicity in a fraction of per $^{0}$ flies (Vosshall and Young, 1995). The observed rescue may reflect PER expression in the adult CRY- and GLASS-expressing DN1s, but our results with the $g l^{60 J} p d f^{01}$ double mutant rule out any contribution of the adult DN1s to the weak (short-period) rhythmicity of $p d f^{01}$ mutants and strains lacking $\mathrm{LN}_{\mathrm{v}} \mathrm{s}$ (Wheeler et al., 1993; Helfrich-Förster, 1998; Renn et al., 1999; Blanchardon et al., 2001). Indeed, in $p d f^{01}$ brains, PER cycling defects were only observed in the DN1s, suggesting that the loss of molecular oscillations in these neurons may participate in the weakening of rhythmicity induced by the absence of PDF signaling from the $\mathrm{LN}_{\mathrm{v}} \mathrm{s}$.

\section{References}

Ahmad M (1999) Seeing the world in red and blue: insight into plant vision and photoreceptors. Curr Opin Plant Biol 2:230-235.

Ahmad M, Grancher N, Heil M, Black RC, Giovani B, Galland P, Lardemer D (2002) Action spectrum for cryptochrome-dependent hypocotyl growth inhibition in Arabidopsis. Plant Physiol 129:774-785.

Aschoff J (1979) Circadian rhythms: influences of internal and external factors on the period measured in constant conditions. Z Tierpsychol 49:225-249.

Bergmann A, Agapite J, McCall K, Steller H (1998) The Drosophila gene hid is a direct molecular target of Ras-dependent survival signaling. Cell 95:331-341.

Blanchardon E, Grima B, Klarsfeld A, Chélot E, Hardin PE, Préat T, Rouyer F (2001) Defining the role of Drosophila lateral neurons in the control of circadian activity and eclosion rhythms by targeted genetic ablation and PERIOD protein overexpression. Eur J Neurosci 13:871-888.

Ceriani MF, Darlington TK, Staknis D, Mas P, Petti AA, Weitz CJ, Kay SA (1999) Light-dependent sequestration of TIMELESS by CRYPTOCHROME. Science 285:553-556.

Ellis MC, O’Neill EM, Rubin GM (1993) Expression of Drosophila glass protein and evidence for negative regulation of its activity in non-neuronal cells by another DNA-binding protein. Development 119:855-865.

Emery P, So WV, Kaneko M, Hall JC, Rosbash M (1998) CRY, a Drosophila clock and light-regulated cryptochrome, is a major contributor to circadian rhythm resetting and photosensitivity. Cell 95:669-679.

Emery P, Stanewsky R, Hall JC, Rosbash M (2000a) A unique circadianrhythm photoreceptor. Nature 404:456-457.
Emery P, Stanewsky R, Helfrich-Förster C, Emery-Le M, Hall JC, Rosbash M (2000b) Drosophila CRY is a deep brain circadian photoreceptor. Neuron 26:493-504.

Fujita SC, Zipursky SL, Benzer S, Ferrus A, Shotwell SL (1982) Monoclonal antibodies against the Drosophila nervous system. Proc Natl Acad Sci USA 79:7929-7933.

Hall JC (2000) Cryptochromes: sensory reception, transduction, and clock functions subserving circadian systems. Curr Opin Neurobiol 10:456-466.

Hamblen M, Zehring WA, Kyriacou CP, Reddy P, Yu Q, Wheeler DA, Zwiebel LJ, Konopka RJ, Rosbash M, Hall JC (1986) Germ-line transformation involving DNA from the period locus in Drosophila melanogaster: overlapping genomic fragments that restore circadian and ultradian rhythmicity to per $^{0}$ and per ${ }^{-}$mutants. J Neurogenet 3:249-291.

Hassan J, Busto M, Iyengar B, Campos AR (2000) Behavioral characterization and genetic analysis of the Drosophila melanogaster larval response to light as revealed by a novel individual assay. Behav Genet 30:59-69.

Hay BA, Wolff T, Rubin GM (1994) Expression of baculovirus P35 prevents cell death in Drosophila. Development 120:2121-2129.

Helfrich-Förster C (1995) The period clock gene is expressed in central nervous system neurons which also produce a neuropeptide that reveals the projections of circadian pacemaker cells within the brain of Drosophila melanogaster. Proc Natl Acad Sci USA 92:612-616.

Helfrich-Förster C (1998) Robust circadian rhythmicity of Drosophila melanogaster requires the presence of lateral neurons: a brain-behavioral study of disconnected mutants. J Comp Physiol [A] 182:435-453.

Helfrich-Förster C, Homberg U (1993) Pigment-dispersing hormoneimmunoreactive neurons in the nervous system of wild-type Drosophila melanogaster and of several mutants with altered circadian rhythmicity. J Comp Neurol 337:177-190.

Helfrich-Förster C, Winter C, Hofbauer A, Hall JC, Stanewsky R (2001) The circadian clock of fruit flies is blind after elimination of all known photoreceptors. Neuron 30:249-261.

Helfrich-Förster C, Edwards T, Yasuyama K, Wisotzky B, Schneuwly S, Stanewsky R, Meinertzhagen IA, Hofbauer A (2002) The extraretinal eyelet of Drosophila: development, ultrastructure, and putative circadian function. J Neurosci 22:9255-9266.

Hofbauer A, Buchner E (1989) Does Drosophila have seven eyes? Z Naturforsch [C] 76:335-336.

Hsu CD, Adams SM, O’Tousa JE (2002) Rpr-and hid-driven cell death in Drosophila photoreceptors. Vision Res 42:507-516.

Ivanchenko M, Stanewsky R, Giebultowicz JM (2001) Circadian photoreception in Drosophila: functions of cryptochrome in peripheral and central clocks. J Biol Rhythms 16:205-215.

Kaneko M (1998) Neural substrates of Drosophila rhythms revealed by mutants and molecular manipulations. Curr Opin Neurobiol 8:652-658.

Kaneko M, Hall JC (2000) Neuroanatomy of cells expressing clock genes in Drosophila: transgenic manipulation of the period and timeless genes to mark the perikarya of circadian pacemaker neurons and their projections. J Comp Neurol 422:66-94.

Kaneko M, Helfrich-Förster C, Hall JC (1997) Spatial and temporal expression of the period and timeless genes in the developing nervous system of Drosophila: newly identified pacemaker candidates and novel features of clock gene product cycling. J Neurosci 17:6745-6760.

Kaneko M, Hamblen MJ, Hall JC (2000) Involvement of the period gene in developmental time-memory: effect of the perShort mutation on phase shifts induced by light pulses delivered to Drosophila larvae. J Biol Rhythms 15:13-30.

Klarsfeld A, Leloup JC, Rouyer F (2003) Analysis of circadian rhythms of locomotor activity in Drosophila. Behav Processes 64:161-175.

Konopka RJ, Pittendrigh C, Orr D (1989) Reciprocal behaviour associated with altered homeostasis and photosensitivity of Drosophila clock mutants. J Neurogenet 6:1-10.

Lin FJ, Song W, Meyer-Bernstein E, Naidoo N, Sehgal A (2001) Photic signaling by cryptochrome in the Drosophila circadian system. Mol Cell Biol 21:7287-7294.

Malpel S, Klarsfeld A, Rouyer F (2002) Larval optic nerve and adult extraretinal photoreceptors sequentially associate with the clock neurons during Drosophila brain development. Development 129:1443-1453.

Malpel S, Klarsfeld A, Rouyer F (2004) Circadian synchronization and rhythmicity in larval photoperception-defective mutants of Drosophila. J Biol Rhythms, in press. 
Moses K, Ellis MC, Rubin GM (1989) The glass gene encodes a zinc-finger protein required by Drosophila photoreceptor cells. Nature 340:531-536.

Nitabach MN, Blau J, Holmes TC (2002) Electrical silencing of Drosophila pacemaker neurons stops the free-running circadian clock. Cell 109:485-495.

Pearn MT, Randall LL, Shortridge RD, Burg MG, Pak WL (1996) Molecular, biochemical, and electrophysiological characterization of Drosophila norpA mutants. J Biol Chem 271:4937-4945.

Renn SC, Park JH, Rosbash M, Hall JC, Taghert PH (1999) A pdfneuropeptide gene mutation and ablation of PDF neurons each cause severe abnormalities of behavioral circadian rhythms in Drosophila. Cell 99:791-802.

Riesgo-Escovar J, Raha D, Carlson JR (1995) Requirement for a phospholipase C in odor response: overlap between olfaction and vision in Drosophila. Proc Natl Acad Sci USA 92:2864-2868.

Shortridge RD, Yoon J, Lending CR, Bloomquist BT, Perdew MH, Pak WL (1991) A Drosophila phospholipase $C$ gene that is expressed in the central nervous system. J Biol Chem 266:12474-12480.

Stanewsky R, Frisch B, Brandes C, Hamblen-Coyle MJ, Rosbash M, Hall JC (1997) Temporal and spatial expression patterns of transgenes contain- ing increasing amounts of the Drosophila clock gene period and a lacZ reporter: mapping elements of the PER protein involved in circadian cycling. J Neurosci 17:676-696.

Stanewsky R, Kaneko M, Emery P, Beretta B, Wager-Smith K, Kay SA, Rosbash M, Hall JC (1998) The cryb mutation identifies cryptochrome as a circadian photoreceptor in Drosophila. Cell 95:681-692.

Suri V, Qian Z, Hall JC, Rosbash M (1998) Evidence that the TIM light response is relevant to light-induced phase shifts in Drosophila melanogaster. Neuron 21:225-234.

Vosshall LB, Young MW (1995) Circadian rhythms in Drosophila can be driven by period expression in a restricted group of central brain cells. Neuron 15:345-360.

Wheeler DA, Hamblen-Coyle MJ, Dushay MS, Hall JC (1993) Behavior in light-dark cycles of Drosophila mutants that are arrhythmic, blind, or both. J Biol Rhythms 8:67-94.

Zhao J, Kilman VL, Keegan KP, Peng P, Emery P, Rosbash M, Allada R (2003) Drosophila clock can generate ectopic circadian clocks. Cell 113: 755-766. 